Check for updates

Cite this: Chem. Sci., 2020, 11, 429

๑ All publication charges for this article have been paid for by the Royal Society of Chemistry

Received 14th June 2019

Accepted 4th November 2019

DOI: $10.1039 / \mathrm{c} 9 \mathrm{sc0} 02911 \mathrm{~g}$

rsc.li/chemical-science

\section{Optical control of the nuclear bile acid receptor FXR with a photohormone $\uparrow$}

\author{
Johannes Morstein, (D) a Julie B. Trads, ${ }^{b}$ Konstantin Hinnah, ${ }^{a}$ Sabine Willems, ${ }^{c}$ \\ David M. Barber, ${ }^{b}$ Michael Trauner, ${ }^{d}$ Daniel Merk (D) ${ }^{c}$ and Dirk Trauner (D)*a
}

\begin{abstract}
Herein, we report a photoswitchable modulator for a nuclear hormone receptor that exerts its hormonal effects in a light-dependent fashion. The azobenzene AzoGW enables optical control of the farnesoid X receptor (FXR), a key regulator of hepatic bile acid, lipid and glucose metabolism. AzoGW was derived from the synthetic agonist GW4064 through an azologization strategy and is a metabolically stable, highly selective photoswitchable FXR agonist in its dark-adapted form. Upon irradiation, the thermally bistable 'photohormone' becomes significantly less active. Optical control of FXR was demonstrated in a luminescence reporter gene assay and through light-dependent reversible transcription modulation of FXR target genes (CYP7A1, Ost $\alpha$, Ost $\beta$ ) in liver cells.
\end{abstract}

Nuclear hormone receptors (NHRs) are ligand-activated transcription factors which interact with DNA and regulate the expression levels of their target genes. ${ }^{1,2}$ As such, they are involved in a broad spectrum of biological processes, ranging from embryonic development, differentiation, homeostasis and metabolism to cell death (endocrine mode of control). A significant portion (ca. 16\%) of all small molecule drugs act on this biological target family, which consists of 48 different receptors in humans. ${ }^{3}$ Endogenous ligands include steroid and thyroid hormones, retinoids, bile acids (BAs), fatty acids, and vitamin D. ${ }^{4}$

The farnesoid $\mathrm{X}$ receptor (FXR) is activated by BAs as endogenous ligands and serves as a central regulator of mammalian metabolism, that controls genes regulating the levels of BAs, lipids, cholesterol, and glucose. ${ }^{5,6}$ It is involved in various physiological and pathophysiological processes, including liver protection and regeneration, inflammation, and carcinogenesis. FXR acts on genes directly involved in BA metabolism and exhibits a number of rapid non-genomic effects, e.g. the control of glucose stimulated insulin secretion and glutamate transport. ${ }^{7}$

\footnotetext{
a Department of Chemistry, New York University, New York, New York 10003, USA. E-mail: dirktrauner@nyu.edu

${ }^{b}$ Department of Chemistry, Center for Integrated Protein Science, Ludwig Maximilians University Munich, 81377 Munich, Germany

'Institute of Pharmaceutical Chemistry, Goethe-University Frankfurt, Max-von-LaueStrasse 9, 60438 Frankfurt, Germany

${ }^{d}$ Hans Popper Laboratory of Molecular Hepatology, Division of Gastroenterology and Hepatology, Department of Internal Medicine III, Medical University of Vienna, Waehringer Guertel 18-20, 1090 Vienna, Austria

$\dagger$ Electronic supplementary information (ESI) available. See DOI: 10.1039/c9sc02911g
}

In recent years, photopharmacology has become a broadly applicable approach to manipulate and study biological systems. ${ }^{8-10}$ While ion channels, GPCRs, enzymes and many other biological targets have been successfully put under reversible optical control with small molecule photoswitches, NHRs have not yet been systematically addressed. Recently, we demonstrated that the azobenzene-containing retinoic acid receptor $\alpha(\operatorname{RAR} \alpha)$ agonist Azo80 ${ }^{11}$ allows for optical control of RAR $\alpha .{ }^{12}$ We envisioned that photoswitchable NHR modulators, termed 'photohormones', could facilitate progress in the study of NHRs. Herein, we describe the development of a photoswitchable FXR agonist, termed AzoGW. This photohormone allows for optical control of FXR as demonstrated through a luminescent reporter gene assay and via transcriptional modulation in a liver cell model system.

AzoGW was derived from the potent FXR agonist GW4064. GW4064 is one of the most widely used FXR agonists with good selectivity over related NHRs. ${ }^{13}$ However, it has recently been shown to exhibit some off-target effects on GPCRs. GW4064 features a trans-stilbene moiety. Replacement of the stilbene with an azobenzene ('azologization') ${ }^{12,14}$ furnished the photoswitchable analog (Fig. 1A), which was synthesized in analogy to its parent compound (Fig. 1B). Diazotization of $m$-aminobenzoic acid and azo-coupling with phenol gave azobenzene $\mathbf{1}$. A subsequent Fischer esterification afforded the corresponding methyl ester 2. In parallel, 2,6-dichlorobenzaldoxime was chlorinated to yield chloro-oxime 3 . Treatment of 3 with 4methyl-3-oxopentanoate under basic conditions then gave isoxazole 4. DIBAL-H reduction yielded primary alcohol 5 , which was coupled with the azobenzene 2 under Mitsunobu conditions. Saponification of the resulting methyl ester then gave AzoGW. 
A<smiles></smiles><smiles>CC(C)c1onc(-c2c(Cl)cccc2Cl)c1Oc1ccc(N=Nc2cccc(C(=O)O)c2)cc1</smiles>
$365 \mathrm{~nm} \downarrow 4600 \mathrm{~nm}$<smiles>CC(C)c1onc(-c2c(Cl)cccc2Cl)c1Oc1ccc(NN2C=C3C(C(=O)OCc4ccccc4)=CC=C32)cc1</smiles>

B<smiles>COC(=O)c1cccc(N=Nc2ccc(O)cc2)c1</smiles>

The photophysical properties of AzoGW were evaluated using UV-Vis spectroscopy (Fig. 2A-D). The UV-Vis spectra of AzoGW after illumination with $\lambda=460 \mathrm{~nm}$ (trans, blue) and $\lambda=$
$365 \mathrm{~nm}$ (cis, gray) demonstrate wavelength-dependent switching as expected for a 'classical' azobenzene (Fig. 2A). Photoswitching could be repeated rapidly over multiple cycles
A

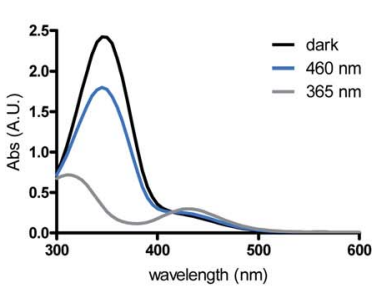

E

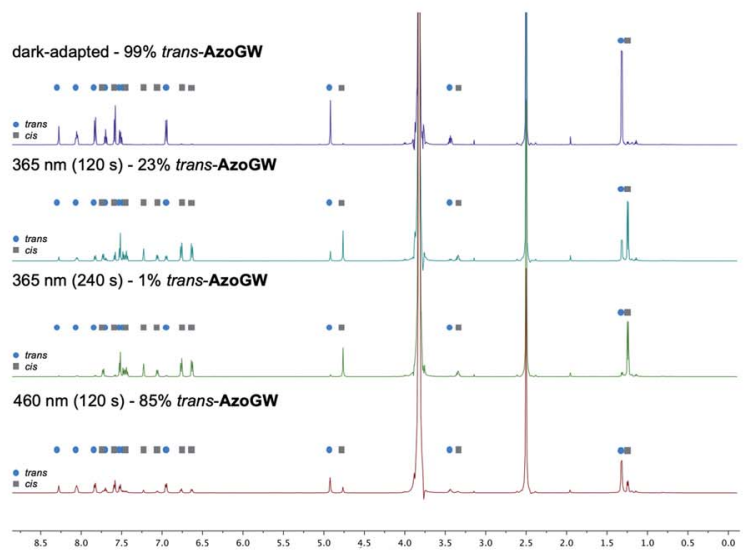

B

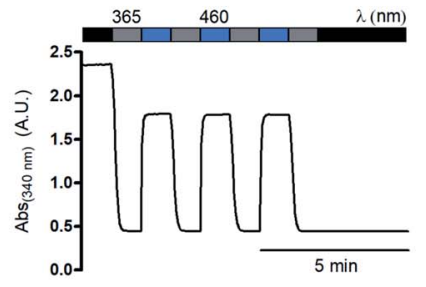

$\mathbf{F}$
D

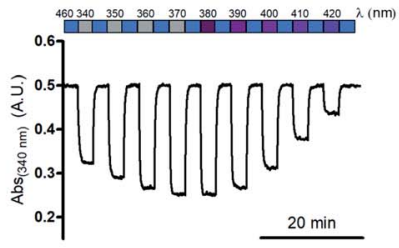

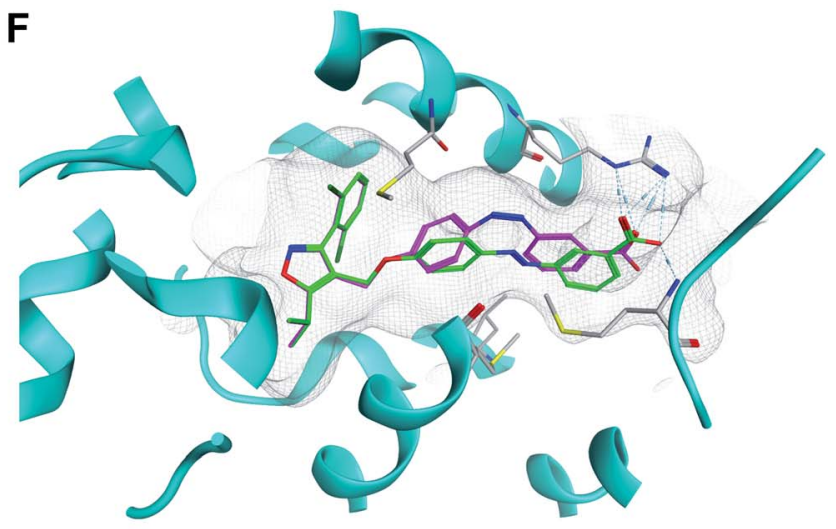

Fig. 2 Photophysical evaluation and molecular docking of AzoGW. (A) The UV-Vis spectra of AzoGW in the dark-adapted (trans, black), 365 nm adapted (cis, gray) and $460 \mathrm{~nm}$ adapted (trans, blue) photostationary states. (B) Reversible cycling between photoisomers with alternating illumination at the two distinct wavelengths $365 \mathrm{~nm}$ and $460 \mathrm{~nm}$, indicating the fast kinetics of the isomerization. (C) Reversible cycling between photoisomers with alternating illumination at varying wavelengths (between $340 \mathrm{~nm}$ and $420 \mathrm{~nm}$ ). (D) AzoGW was repeatedly switched between its cis- and trans-state for 50 cycles. (E) ${ }^{1} \mathrm{H}$-NMR-studies of AzoGW in the dark-adapted, $365 \mathrm{~nm}$ adapted and $460 \mathrm{~nm}$ adapted photostationary states in DMSO- $d_{6} / D_{2} \mathrm{O}$. (F) Molecular docking of trans-AzoGW (green) and cis-AzoGW (purple) to the FXR ligand binding site (PDB-ID: 3DCT15). 
A

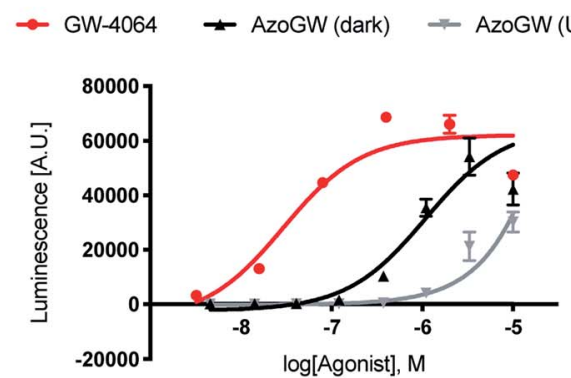

C

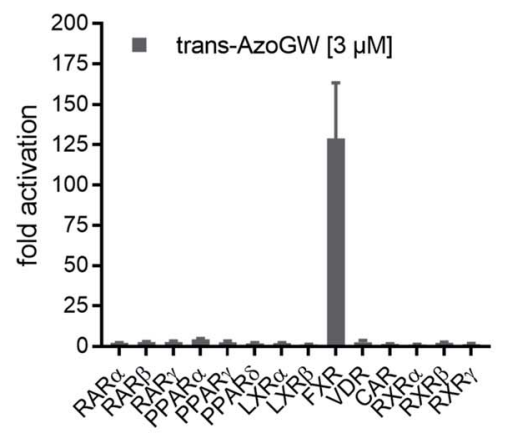

B

D<smiles>CC=CC=C=S</smiles>
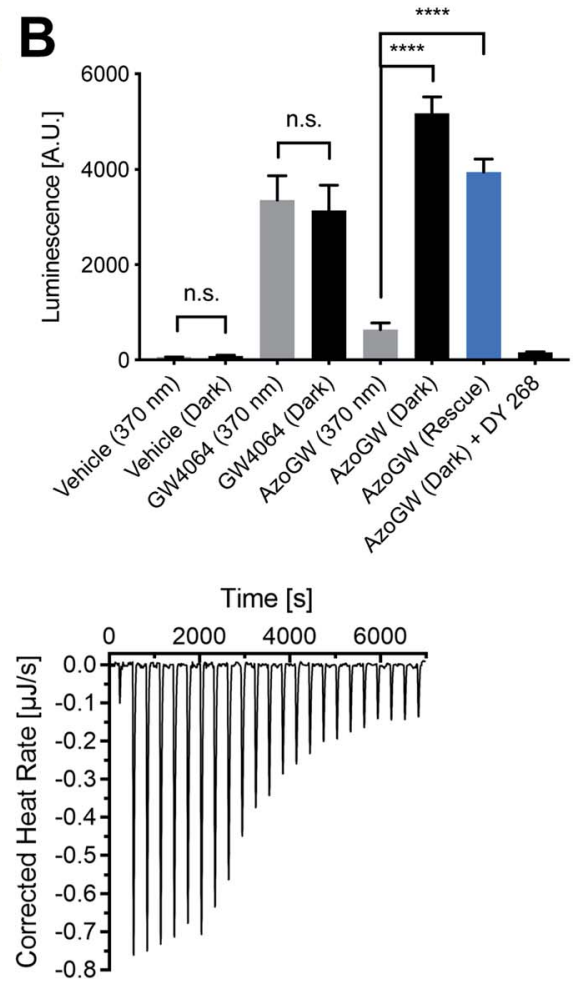

$\mathbf{E}$

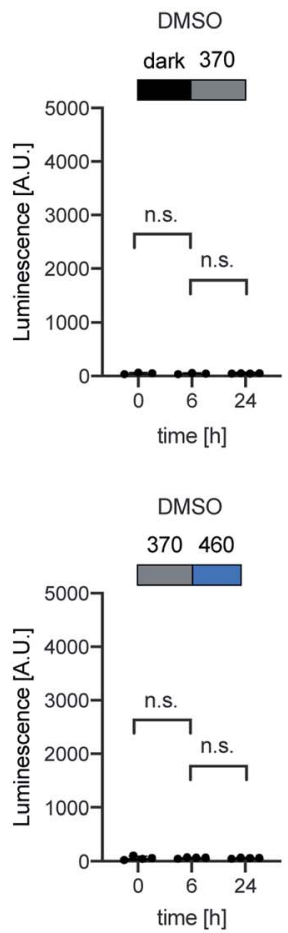

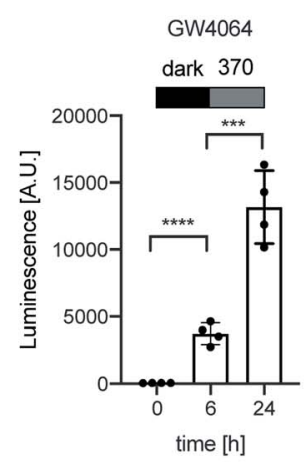

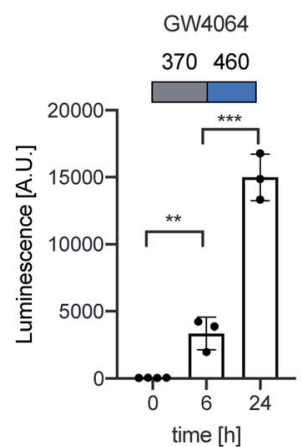

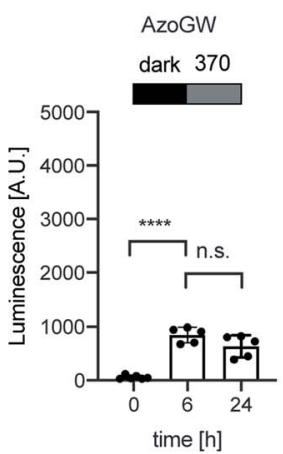

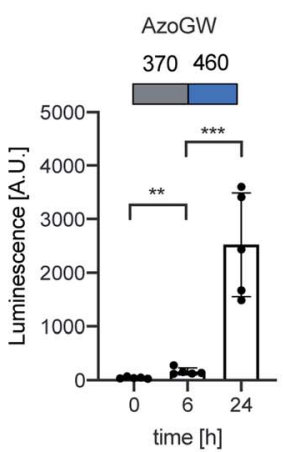

Fig. 3 Pharmacological evaluation of AzoGW. (A) Dose responses of GW4064, trans-AzoGW and cis-AzoGW in a luminescent reporter cell line after $22 \mathrm{~h}$. Samples were run in duplicate and in two independent experiments. Error bars represent mean \pm SD (B) control and rescue (reversibility) experiments using GW4064 (41 nM), AzoGW (367 nM), and DY $268(2 \mu \mathrm{M})$. Samples were run at least in triplicate. Error bars represent SEM ${ }^{* * *} p<0.001$, n.s., not significant, Student's $t$-test. (C) Selectivity panel for trans-AzoGW $(3 \mu M)$ over several related nuclear hormone receptors. Samples were run in duplicate and in four independent experiments. Error bars represent SEM. (D) Isothermal titration calorimetry (ITC) experiment with recombinant FXR LBD and AzoGW (dark-adapted, $15 \mu \mathrm{M}$ ). $K_{\mathrm{D}}=433 \mathrm{~nm}$. (E) Bidirectional switching experiment with AzoGW in luminescent reporter cell line using GW4064 $(0.5 \mu \mathrm{M})$ and AzoGW $(2 \mu \mathrm{M})$. To switch from dark to $370 \mathrm{~nm}$ cells were removed from the dark after 6 hours, irradiated with $365 \mathrm{~nm}$ for 3 minutes and subsequently irradiated with the Cell DISCO (370 nm) system for $75 \mathrm{~ms}$ every $15 \mathrm{~s}$. To switch from $365 \mathrm{~nm}$ cells were removed from the Cell DISCO (370 nm) system after 6 hours, irradiated at $465 \mathrm{~nm}$ for 3 minutes and incubated in the dark until $24 \mathrm{~h}$. 
(Fig. 2B), the amount of active photoswitch could be titrated with different wavelengths of light ('color-dosing', Fig. 2C), and no photobleaching, photodegradation, or fatigue was observed after numerous switching cycles (Fig. 2D). cis-AzoGW exhibits a thermal relaxation half-life time of $52 \mathrm{~h}$ at room temperature in physiological buffer (ESI Fig. S1 $\dagger$ ) and can thus be classified as a bistable photoswitch.

In some cases, the metabolic stability of azobenzenes is compromised by reductive cleavage by bacteria or glutathione (GSH) reduction. ${ }^{16}$ To assess the metabolic stability of AzoGW, we assayed its stability in the presence of the bacterial enzyme azoreductase in $E$. coli (ESI Fig. S5 $†$ ). We found that AzoGW exhibits excellent stability whilst methyl red is degraded over time. Additionally, we evaluated the stability of AzoGW in the presence of GSH (ESI Fig. S6 $†$ ). Although GSH enhances the thermal relaxation of cis-AzoGW, we did not observe notable degradation, supporting that AzoGW is metabolically stable which makes it potentially useful for applications in cultured cells or in vivo.

We further investigated the binding mode of AzoGW by molecular docking to a co-crystal of GW4064 and FXR (PDB-ID: $3 \mathrm{DCT}^{\mathbf{1 5}}$ ). The docking pose of trans-AzoGW matches the crystallized binding mode of GW4064 (ESI Fig. S7 $\dagger$ ). The cis-isomer of AzoGW deviates considerably from the binding pose of GW4064 and trans-AzoGW and the carboxylic acid group is less close to engaging residues (Fig. 2E). These results suggest reduced potency of the cis-isomer in keeping with the predictions of our chemoinformatic azologization study. ${ }^{\mathbf{1 2}}$

To explore if AzoGW allows for light-dependent activation of FXR, we carried out a luminescent reporter gene assay for human FXR, where the ligand-binding dependent activation of FXR is coupled to the expression of the reporter gene firefly luciferase. We used varying concentrations of GW4064 and AzoGW (Fig. 3A). A Cell DISCO system was employed to achieve switching of trans-AzoGW to cis-AzoGW (370 nm, $75 \mathrm{~ms}$ pulse every $15 \mathrm{~s}$ ). ${ }^{17}$ We found that trans-AzoGW (dark, EC $_{50}=1.1 \times$ $10^{-6} \mathrm{M}$ ) is approximately 100 -fold more potent than the less active cis-isomer of AzoGW (UV, $\mathrm{EC}_{50}=9.5 \times 10^{-5} \mathrm{M}$ ). These results demonstrate that good optical control of FXR activation could be obtained with this tool at low micromolar concentrations. As a control experiment, we showed that light does not affect transcription levels (Fig. 3B). Moreover, the FXR antagonist DY $268(2 \mu \mathrm{M})$ inhibits the effects of trans-AzoGW (367 nM) demonstrating target-specificity in our assay. A rescue experiment demonstrated reversibility of the reported effects. To this end, AzoGW (367 nM) was added to cells as $365 \mathrm{~nm}$-adapted cisAzoGW and after 5 min the cells were illuminated with $460 \mathrm{~nm}$ light for $2 \mathrm{~min}$ to reactivate AzoGW. Similar levels of transcription were observed compared to the experiment with darkadapted trans-AzoGW (Fig. 3B). In a hybrid reporter gene assay for the nuclear hormone receptors $\operatorname{PPAR} \alpha / \gamma / \delta, \operatorname{RXR} \alpha / \beta / \gamma, \operatorname{LXR} \alpha /$ $\beta, \mathrm{RAR} \alpha / \beta / \gamma, \mathrm{FXR}, \mathrm{VDR}$, and CAR, we verified that trans-AzoGW selectively activates FXR over other related receptors (Fig. 3C).

Next, we analyzed the capacity of AzoGW for the optical control of FXR-dependent gene expression in untransfected liver cells (HepG2). To this end, we analyzed expression levels of CYP7A1 as well as Ost $\alpha / \beta$ mRNA. CYP7A1 encodes a monooxygenase that accounts for the rate-limiting step in bile acid biosynthesis. Ost $\alpha$ and Ost $\beta$ encode bile acids transporters
A

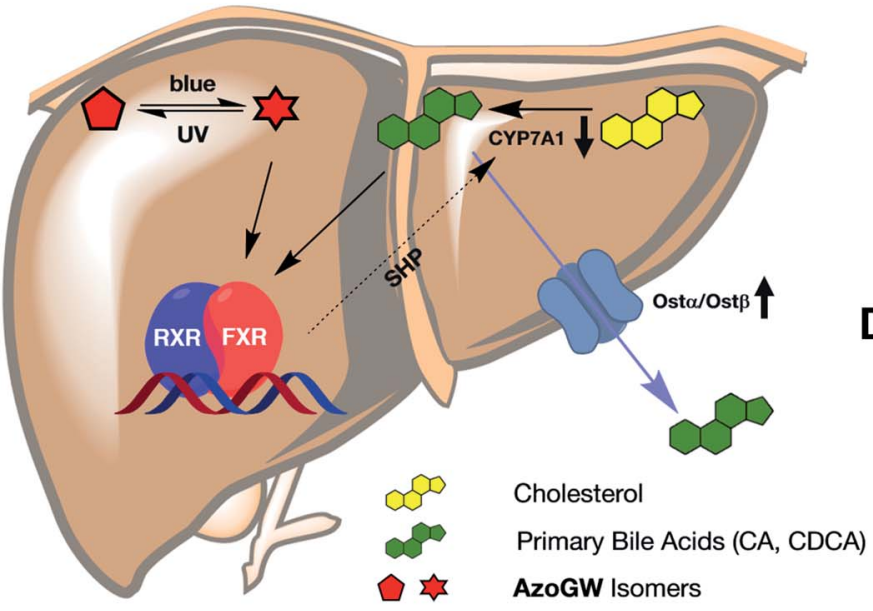

B

D
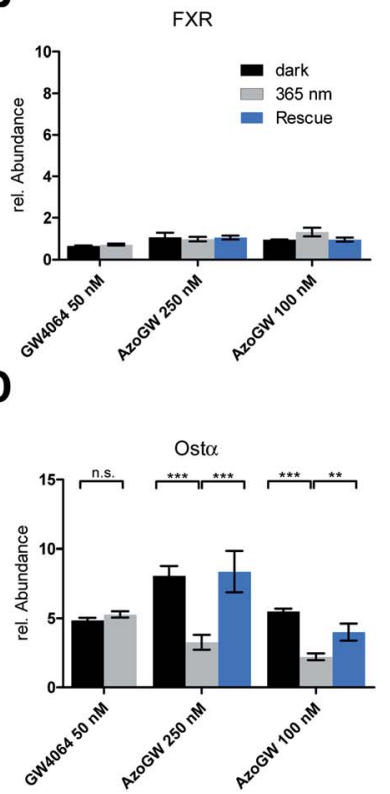

C

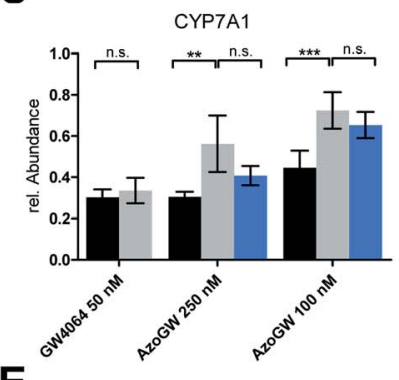

E

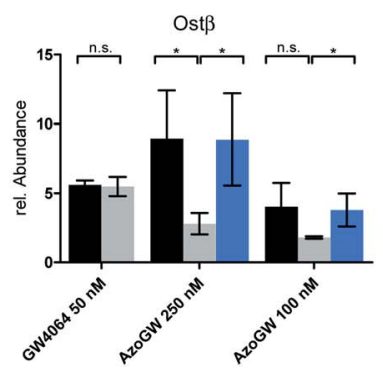

Fig. 4 (A) Role of FXR in physiology: Agonist induced activation of FXR, either by its endogenous ligands (BA) or synthetic ligands (GW4064, AzoGW), leads to an up-or downregulation of its target genes. FXR indirectly suppresses CYP7A1 which catalyzes the rate determining step in BA biosynthesis from cholesterol. Also, FXR promotes expression levels of the BA export pumps Ost $\alpha$ and Ost $\beta$. (B-E) Effects of GW4064 and AzoGW on FXR. Depicted are the mRNA expression levels of FXR, CYP7A1, Ost $\alpha$ and Ost $\beta$, relative to the vehicle control (1\% DMSO) after incubation for $24 \mathrm{~h}$ in the dark (black) or under illumination with $\lambda=365 \mathrm{~nm}$ (gray) and $\lambda=460 \mathrm{~nm}$ (blue) respectively. Samples were run in three independent experiments. Error bars represent SEM $* * * p<0.001, * * p<0.01, * p<0.1$, n.s., not significant, Student's $t$-test. 
(Fig. 4A). As expected, treatment of HepG2 cells with GW4064 resulted in a robust suppression of CYP7A1, whereas a strong upregulation of Ost $\alpha$ and Ost $\beta$ was observed (Fig. 4C-E). Expression levels of FXR remained unaffected (Fig. 4B). We also saw no differences in the light-excluded (dark) and irradiated samples $(365 \mathrm{~nm})$ with GW4064, confirming that light at the intensities used in our experiments does not mediate changes in FXR-dependent transcription levels.

Next, we investigated whether AzoGW could put FXR dependent gene expression under optical control. Indeed, incubation of HepG2 cells with different concentrations of AzoGW showed light-dependent suppression of CYP7A1 and upregulation of Ost $\alpha$ and Ost $\beta$ (Fig. 4C-E). As expected, transAzoGW is a more potent agonist of FXR, leading to a stronger modulation of FXR target genes. Relative abundance of FXR did not change significantly. This supports that the observed expression level modulations exclusively originate in differences in the effect of trans-AzoGW and cis-AzoGW on FXR. A rescue experiment was conducted to assess the reversibility of the inhibitory and promoting effect of AzoGW (Fig. 4, blue bars) concluding that illumination with blue light $(\lambda=460 \mathrm{~nm})$ significantly restores the activity of trans-AzoGW (referred to as the dark-adapted state).

In summary, we report the development of a photohormone for the nuclear hormone receptor FXR. AzoGW exhibits excellent photo- and metabolic stability and allows for photocontrol of FXR. Most importantly, FXR-dependent transcription was brought under optical control in an untransfected liver cell line, demonstrating the potential of this tool in cellular systems. Our study underscored that optogenetic and photopharmacological technologies can be applied to nuclear receptors, which include thyroid hormone receptors (TRs), peroxisome proliferatoractivated receptors (PPARs), and estrogen receptors (ERs). In addition, to azologization strategies, many NHRs could potentially be addressed through the design of photoswitchable amphiphilic (lipid-like) agonists. ${ }^{18-20}$ Photohormones could enable precise genomic regulation in complex signaling networks and rapid control of non-genomic NHR effects. As such, they could lead to new insights into NHR physiology and find therapeutic applications using endoscopic/endoluminal light delivery. ${ }^{21,22}$

\section{Conflicts of interest}

M. T. served as a speaker and/or consultant for Albireo, Boehringer Ingelheim, BiomX, Falk, Gilead, Intercept, Novartis, Phenex, Regulus and Shire, and received travel support from Falk, Gilead, and Intercept, as well as grants/research support from Albireo, Cymabay, Falk, Gilead and Intercept. He is also co-inventor of patents on the medical use of 24-norursodeoxycholic acid.

\section{Acknowledgements}

J. M. and K. H. thank the German Academic Scholarship Foundation for a fellowship and J. M. thanks the New York University for a MacCracken fellowship and a Margaret and
Herman Sokol fellowship. J. B. T. thanks the Danish National Research Foundation Center for DNA Nanotechnology (DNRF81) and Aarhus University for financial support. D. M. B. thanks the European Commission for a Marie SkłodowskaCurie Intra-European Fellowship (PIEF-GA-2013-627990). We thank Dr Julia Ast, Dr David Hodson, and Dr Thierry Claudel for experimental support in the initial stages of the project and helpful discussion. We thank Dr Christian Fischer, Christopher J. Arp, Grace Pan and Alan Liu for insightful discussion and proofreading of the manuscript.

\section{References}

1 R. M. Evans and D. J. Mangelsdorf, Nuclear Receptors, RXR, and the Big Bang, Cell, 2014, 157(1), 255-266, DOI: 10.1016/ j.cell.2014.03.012.

2 H. Gronemeyer, J.-Å. Gustafsson and V. Laudet, Principles for Modulation of the Nuclear Receptor Superfamily, Nat. Rev. Drug Discovery, 2004, 3(11), 950-964, DOI: 10.1038/ nrd1551.

3 R. Santos, O. Ursu, A. Gaulton, A. P. Bento, R. S. Donadi, C. G. Bologa, A. Karlsson, B. Al-Lazikani, A. Hersey, T. I. Oprea, et al., A Comprehensive Map of Molecular Drug Targets, Nat. Rev. Drug Discovery, 2017, 16(1), 19-34, DOI: 10.1038/nrd.2016.230.

4 Overview of Nomenclature of Nuclear Receptors|Pharmacological Reviews, http:// pharmrev.aspetjournals.org/content/58/4/685.long, accessed Dec 10, 2018.

5 F. Y. Lee, H. Lee, M. L. Hubbert, P. A. Edwards and Y. Zhang, FXR, a Multipurpose Nuclear Receptor, Trends Biochem. Sci., 2006, 31(10), 572-580, DOI: 10.1016/j.tibs.2006.08.002.

6 Y.-D. Wang, W.-D. Chen, D. D. Moore and W. Huang, FXR: A Metabolic Regulator and Cell Protector, Cell Res., 2008, 18(11), 1087-1095, DOI: 10.1038/cr.2008.289.

7 B. Renga, A. Mencarelli, P. Vavassori, V. Brancaleone and S. Fiorucci, The Bile Acid Sensor FXR Regulates Insulin Transcription and Secretion, Biochim. Biophys. Acta, Mol. Basis Dis., 2010, 1802(3), 363-372, DOI: 10.1016/ j.bbadis.2010.01.002.

8 K. Hüll, J. Morstein and D. Trauner, In Vivo Photopharmacology, Chem. Rev., 2018, 118(21), 1071010747, DOI: 10.1021/acs.chemrev.8b00037.

9 W. A. Velema, W. Szymanski and B. L. Feringa, Photopharmacology: Beyond Proof of Principle, J. Am. Chem. Soc., 2014, 136(6), 2178-2191, DOI: 10.1021/ ja413063e.

10 A. A. Beharry and G. A. Woolley, Azobenzene Photoswitches for Biomolecules, Chem. Soc. Rev., 2011, 40(8), 4422-4437, DOI: 10.1039/C1CS15023E.

11 H. Kagechika, T. Himi, K. Namikawa, E. Kawachi, Y. Hashimoto and K. Shudo, Retinobenzoic Acids. 3 Structure-Activity Relationships of Retinoidal Azobenzene4-Carboxylic Acids and Stilbene-4-Carboxylic Acids, J. Med. Chem., 1989, 32(5), 1098-1108, DOI: 10.1021/jm00125a027.

12 J. Morstein, M. Awale, J.-L. Reymond and D. Trauner, Mapping the Azolog Space Enables the Optical Control of 
New Biological Targets, ACS Cent. Sci., 2019, 5(4), 607-618, DOI: 10.1021/acscentsci.8b00881.

13 N. Singh, M. Yadav, A. K. Singh, H. Kumar, S. K. D. Dwivedi, J. S. Mishra, A. Gurjar, A. Manhas, S. Chandra, P. N. Yadav, et al., Synthetic FXR Agonist GW4064 Is a Modulator of Multiple G Protein-Coupled Receptors, Mol. Endocrinol., 2014, 28(5), 659-673, DOI: 10.1210/me.2013-1353.

14 J. Broichhagen, J. A. Frank and D. Trauner, A Roadmap to Success in Photopharmacology, Acc. Chem. Res., 2015, 48(7), 1947-1960, DOI: 10.1021/acs.accounts.5b00129.

15 A. Akwabi-Ameyaw, J. Y. Bass, R. D. Caldwell, J. A. Caravella, L. Chen, K. L. Creech, D. N. Deaton, S. A. Jones, I. Kaldor, Y. Liu, et al., Conformationally Constrained Farnesoid X Receptor (FXR) Agonists: Naphthoic Acid-Based Analogs of GW 4064, Bioorg. Med. Chem. Lett., 2008, 18(15), 43394343, DOI: 10.1016/j.bmcl.2008.06.073.

16 Z. B. Mehta, N. R. Johnston, M.-S. Nguyen-Tu, J. Broichhagen, P. Schultz, D. P. Larner, I. Leclerc, D. Trauner, G. A. Rutter and D. J. Hodson, Remote Control of Glucose Homeostasis in Vivo Using Photopharmacology, Sci. Rep., 2017, 7(1), 291, DOI: 10.1038/s41598-017-00397-0.

17 M. Borowiak, W. Nahaboo, M. Reynders, K. Nekolla, P. Jalinot, J. Hasserodt, M. Rehberg, M. Delattre, S. Zahler, A. Vollmar, et al., Photoswitchable Inhibitors of Microtubule Dynamics Optically Control Mitosis and Cell
Death, Cell, 2015, 162(2), 403-411, DOI: 10.1016/ j.cell.2015.06.049.

18 J. A. Frank, M. Moroni, R. Moshourab, M. Sumser and G. R. Lewin, Photoswitchable Fatty Acids Enable Optical Control of TRPV1, Nat. Commun., 2015, 6, 7118, DOI: 10.1038/ncomms8118.

19 J. A. Frank, D. A. Yushchenko, D. J. Hodson, N. Lipstein, J. Nagpal, G. A. Rutter, J.-S. Rhee, A. Gottschalk, N. Brose, C. Schultz, et al., Photoswitchable Diacylglycerols Enable Optical Control of Protein Kinase C, Nat. Chem. Biol., 2016, 12(9), 755-762, DOI: 10.1038/nchembio.2141.

20 J. Morstein, R. Z. Hill, A. J. E. Novak, S. Feng, D. D. Norman, P. C. Donthamsetti, J. A. Frank, T. Harayama, B. M. Williams, A. L. Parrill, et al., Optical Control of Sphingosine-1Phosphate Formation and Function, Nat. Chem. Biol., 2019, 15(6), 623, DOI: 10.1038/s41589-019-0269-7.

21 M. M. Lerch, M. J. Hansen, G. M. van Dam, W. Szymanski and B. L. Feringa, Emerging Targets in Photopharmacology, Angew. Chem., Int. Ed., 2016, 55(37), 10978-10999, DOI: 10.1002/anie.201601931.

22 J. Morstein and D. Trauner, New Players in Phototherapy: Photopharmacology and Bio-Integrated Optoelectronics, Curr. Opin. Chem. Biol., 2019, 50, 145-151, DOI: 10.1016/ j.cbpa.2019.03.013. 